\title{
Probióticos y Prebióticos. Rol en la Terapéutica de la Enfermedad Diarreica Aguda Infantil
}

\author{
Probiotics and Prebiotics. Its Role in Childhood Acute Diarrheal Disease Therapy
}

Edgar Játiva-Mariño ${ }^{1,2}$; Carlos Manterola ${ }^{1,3,4}$; Roque Macias $^{2,5}$ \& Daniel Narváez ${ }^{6}$

JÁTIVA-MARIÑO, E.; MANTEROLA, C.; MACIAS, R. \& NARVÁEZ, D. Probióticos y prebióticos. Rol en la terapéutica de la enfermedad diarreica aguda infantil. Int. J. Morphol., 39(1):294-301, 2021.

RESUMEN: La enfermedad diarreica aguda infantil (EDAI), constituye un problema de salud pública, representando la $2^{\mathrm{a}}$ causa de morbimortalidad infantil en menores de 5 años, en el Ecuador. La hidratación oral y parenteral en los niños hospitalizados bajo normas de administración de conformidad con el grado de deshidratación y pérdida de peso, así como medidas preventivas como la vacunación obligatoria contra el rotavirus, han contribuido a disminuir, pero no a solucionar este problema de salud infantil. Múltiples factores contribuyen para que no se resuelva: socioeconómicos, educacionales, el destete temprano y malas prácticas alimenticias, entre otros. Últimos estudios han propuesto la utilización de probióticos que contribuyan a disminuir el problema sugieriendo el usode Saccharomyces boulardii (SB), asociado a un prebiótico; lo que permitiría acortar el tiempo de tratamiento de una EDAI; por lo que la simbiosis entre SB y un prebiótico denominado fructooligosacárido (FOS), podría ser una alternativa para reducir costos y complicaciones. Una alternativa para medir el curso clínico de una EDAI en infantes es la escala BITTS, de reciente creación y fácil aplicación por clínicos. El objetivo de este manuscrito fue resumir la evidencia existente respecto del rol de losprobióticos y prebióticos en la terapéutica de de la EDAI.

PALABRAS CLAVE: Saccharomyces bourdali; Prebióticos; Probióticos; Enfermedad diarreica aguda; Escala de BITSS.

\section{INTRODUCCIÓN}

Se estima que en sus primeros 5 años de vida, aproximadamente 17 de cada 100 niños sufre un episodio de diarrea; 9 buscan atención médica; uno de cada 20 necesitan ser hospitalizados; y 1 de cada 375 mueren a causa de esto. De hecho, en el registro de 2017 se verificó que entre las causas demortalidaden menores de 5 años, la enfermedad diarreica aguda infantil (EDAI) representó el 2,6 \% (Instituto Nacional de Estadística y Censos, 2017).

Existe evidencia que en el hospital de niños Baca Ortiz, la prevalencia de rotavirus en un corte realizado en 2017 , fue $35,8 \%$; afectando a 1 de cada 3 niños menores de 5 años; con una tasa de mortalidad de 4,0 por mil niños. El tiempo de estancia hospitalaria promedio fue de 2,0 $\pm 0,8$ días; con costes promedios de hospitalización y total por niño de U\$208 y U\$416 por día respectivamente (Instituto Nacional de Estadística y Censos).
En el avance terapéutico, señala que la asociación de un probiótico de la cepa de levaduras Saccharomyces boulardii (SB) y un fructooligosacárido (FOS), podrían potenciar su acción terapéutica en pacientes con EDAI (Bertelsen et al., 2016). Y una alternativa para medir el curso clínico de una EDAI en infantes, es la escala BITTS (Vandenplas et al., 2017), de reciente creación y fácil aplicación por clínicos.

El objetivo de este manuscrito fue resumir la evidencia existente respecto del rol de los probióticos y prebióticos en la terapéutica de de la EDAI; focalizándose en la aplicación de la escala BITTS y del tratamiento asociado de SB y FOS.

\section{ECOLOGÍA INTESTINAL}

La microbiota intestinal en los primeros años de vida

\footnotetext{
${ }^{1}$ Programa de Doctorado en Ciencias Médicas, Universidad de La Frontera, Temuco, Chile.

${ }^{2}$ Universidad Central del Ecuador.

${ }^{3}$ Departamento de Cirugía, Universidad de La Frontera, Temuco, Chile.

${ }^{4}$ Centro de Excelencia en Estudios Morfológicos y Quirúrgicos (CEMyQ), Universidad de La Frontera, Temuco, Chile.

${ }^{5}$ Hospital Pedátrico Baca Ortiz, Quito, Ecuador.

${ }^{6}$ Pontificia Universidad Católica del Ecuador.
} 
se desarrolla igual que los sistemas cognositivos, inmunológicos y otros en el organismo. Es dinámica y tiene influencia de varios factores, como el tipo de nacimiento, la edad gestacional al nacer, si es de término o pretérmino; y el tipo de alimentación si es por lactancia materna o uso de leches "maternizadas" Se considera que la leche humana guía la microbiota y la presencia de oligosacáridos en la leche materna estimula una microbiota intestinal que favorece la inducción de tolerancia (Wopereis et al., 2018).

La microbiota intestinal constituye un complejo ecosistema integrado por más de 400 especies bacterianas, comienza a adquirirse después del nacimiento, Se ha llegado a considerar que en el cuerpo humano existen aproximadamente 10 bacterias por cada célula humana (Foster \& McVey Neufeld, 2013); y tanto la velocidad de colonización como el tipo de microrganismos tienen gran repercusión en el desarrollo del sistema inmune, la regulación de la permeabilidad y el mantenimiento del equilibrio intestinal (Hernández Hernández et al., 2015). Dicho proceso se considera determinante de la susceptibilidad a las infecciones microbianas y de la sensibilidad a los antígenos o alérgenos de la dieta, especialmente durante los primeros años de vida (Bernardo, 2013). Inicialmente, existe un predominio de microrganismos aerobios o anaerobios facultativos (Enterobacteriaceae y Lactobacillus); los que son desplazados posteriormente por bacterias anaerobias estrictas (Bifidobacterium, Bacteroides, Eubacterium, etc.), hasta que se establece una flora prácticamente definitiva (Bernardo). Por el contrario, el intestino de niños alimentados con fórmulas lácteas infantiles está colonizado por una microbiota más heterogénea, caracterizada por colonias de Enterobacteriaceae, Bacteroides, Clostridium, Streptococcus, etc. (Montrose \& Floch, 2005). La menor incidencia de infecciones gastrointestinales, así como de otras enfermedades en niños alimentados con lactancia materna se ha relacionado con su influencia en la composición de la microbiota intestinal, dominada hasta en un $91 \%$ por gérmenes no patógenos del género Bifidobacterium (Agostoni et al., 2004; Montrose \& Floch).

Por el contrario, el intestino de niños alimentados con fórmulas lácteas infantiles está colonizado por una microbiota más heterogénea, caracterizada por colonias de Enterobacteriaceae, Bacteroides, Clostridium, Streptococcus, etc. (Montrose \& Floch).

Existe evidencia que demuestra que la microbiota vaginal de la madre no se relaciona estrechamente con la microbiota fecal del recién nacido; y la lactancia materna se ha mostrado como el principal factor protector en la iniciación, desarrollo y composición de la microbiota del niño, ya que es una fuente continua de bacterias comensales y mutualistas para el intestino del lactante, llegando a contener más de 104 UFC/mL, especialmente de Streptococcus, enterococos, staphylococcus y Lactobacillus (L. acidophilus, L. casei, L. fermentum, L. gasseri, L. johnsonii, L. paracasei, L. plantarum, L. reuteri, L. rhamnosus, L. salivarius, etc.) (Khanna \& Tosh, 2014).

\section{ROL DE LA MICROBIOTA EN EL TRACTO INTES- TINAL INFANTIL}

Las células del epitelio intestinal, el moco que cubre la mucosa, y el flujo sanguíneo que la irriga y las secreciones (fosfolípidos, bilis, péptidos antimicrobianos, etc.); constituyen de forma conjunta una barrera física y química que contribuye a la defensa del huésped (Sanz et al., 2006).

La evidencia actual apoya el papel de lamicrobiota en la promoción y el mantenimiento de una respuesta inmune equilibrada y el establecimiento de la barrera intestinal en la vida postnatal inmediata. El cuerpo humano alberga una población microbiana dinámica y compleja, que incluye alrededor de 500-1,000 especies diferentes. En el período perinatal, los recién nacidos están expuestos no solo a una gran diversidad microbiana, sino también a una variedad de organismos como virus, hongos y parásitos. Después del destete, el intestino del bebé es colonizado por una microbiota que se diversifica rápidamente y conduce a un patrón de flora intestinal similar al de un adulto (Collado et al., 2015).

El moco está integrado por mucinas (glucoproteínas), que son potenciales sitios de adhesión para las bacterias. La síntesis y la composición de las mucinas están reguladas genéticamente en cada individuo. Además, las bacterias intestinales pueden contribuir a la regulación del repertorio de mucinas mediante la modificación de la expresión génica de glucosiltransferasas del huésped y por acción de sus propias enzimas glucolíticas (Macfarlane et al., 2008).

Los patógenos normalmente alteran la permeabilidad intestinal, mientras que las bacterias comensales beneficiosas y los probióticos pueden contribuir al restablecimiento de ésta y de las uniones intercelulares, y favorecer la proliferación celular. La síntesis de péptidos antimicrobianos (defensinas) y proteasas implicadas en su activación en las células de Paneth, constituyen un mecanismo adicional de defensa del huésped frente a agentes patógenos.

Tanto la producción de las defensinas como de las enzimas que las activan puede ser modulada por el microbiota comensal y por bacterias probióticas. Por el contrario, algunos patógenos o agentes oportunistas pueden desarrollar mecanismos de resistencia a esta barrera mediante la reducción de la expresión de dichos péptidos (Macfarlane et al.; Grönlund et al., 2011). 
El sistema inmune de la mucosa intestinal. Este, es el órgano inmunológico principal del organismo. Se encuentra formado por tejido linfoideo como los nódulos linfáticos mesentéricos y los nódulos agregados de íleon (placas de Peyer) y por linfocitos difusamente distribuidos a través de la lámina propia y el epitelio. Es capaz de reconocer determinados agentes exógenos como componentes estructurales o toxinas microbianas; así como de secretar mediadores celulares, como inmunoglobulinas, y citocinas, responsables del desencadenamiento de la respuesta inmune. Además hoy cada día se está aclarando el papel importante de la comunicación microbiota-cerebro y está emergiendo como un factor en el desarrollo y la función del cerebro (Foster, 2016).

Por otra parte, la secreción de inmunoglobulina A (IgA), es una de las primeras defensas de la mucosa frente a microrganismos patógenos y su inducción los nódulos agregados de íleon y la lámina propia depende de su interacción con el microbiota comensal.

Asimismo, el tejido linfoide y las células epiteliales poseen receptores (toll-like receptors), que son capaces de reconocer ciertas moléculas bacterianas, entre las que se encuentran polisacáridos, ácidos teicoicos y secuencias de ADN CpG no metiladas.

Las bacterias generan mecanismos protectores mediante la formación de una barrera secretora para evitar que las bacterias patógenas entren en contacto con la superficie de los enterocitos, y una barrera física por medio de una capa de moco epitelial. También aumentan la resistencia de la mucosa intestinal a la colonización con microorganismos patógenos (Zamudio-Vázquez et al., 2017).

El mecanismo por el cual el huésped es capaz de discriminar entre el microbiota comensal y la patógena se desconoce, pero se supone que existen distintas secuencias señal en los dos grupos de microrganismos cuyo reconocimiento por el huésped desencadena a su vez, diversas respuestas (proinflamatorias o no). El microbiota comensal se considera implicado en el desarrollo de la estructura y la funcionalidad del epitelio intestinal y, especialmente, de la inmunidad celular y humoral durante el periodo neonatal (Allen et al., 2010).

Disbiosis. Se refiere a un desequilibrio de bacterias intestinales que ocupan la luz del intestino, a cambios adversos en los patrones de microbiomas que se han asociado con el desarrollo y la progresión de la enfermedad. Se ha informado además que la disbiosis es objeto de múltiples delineaciones explícitas y semi-explícitas. Las enfermedades crónicas que se han asociado con la disbiosis del microbioma intestinal incluyen enfermedades inflamatorias intestinales (p. Ej., Colitis ulcerosa, síndrome del intestino irritable), enfermedades autoinmunes ( $p$. Ej., Esclerosis múltiple, asma),enfermedades metabólicas (p. Ej., Diabetes), neurodegenerativas enfermedades (p. ej., enfermedad de Parkinson, demencias) y cáncer (Vitetta et al., 2018).

El eje intestino cerebro. Este fundamental eje que se ha profundizado su estudio cada día nos da explicaciones de varias enfermedades que se explican en la relación neuroendocrina y al componente neuronal del intestino, considerándose su amplia extensión y el número de neuronas que se encuentran distribuidas a lo largo del sistema intestinal. Las conexiones vías aferentes y eferentes a través del sistema simpático y parasimpático forman la red de este valioso eje que cada día se lo estudia más.

El microbiota intestinal es un regulador clave del eje intestino-cerebro. El intestino es el hogar de una gran variedad de billones de microbios, principalmente bacterias, pero también arqueas, levaduras, parásitos helmintos, virus y protozoos (Gaci et al., 2014).

Varios estudios en diseño experimental han demostrado la sugerencia de que la microbiota intestinal influyen en el estrés, comportamiento, incluidos la ansiedad y la depresión (Foster et al., 2017).

El complejo sistema de comunicación entre el intestino y el cerebro no solo garantiza el mantenimiento y la coordinación adecuados de las funciones gastrointestinales para apoyar el comportamiento y los procesos fisiológicos, sino que también permite que la retroalimentación del intestino ejerza efectos profundos sobre el estado de ánimo, el comportamiento motivado y las funciones cognitivas superiores. La microbiota intestinal se ha convertido en un componente crítico que afecta potencialmente a todas estas vías neuroinmunoendocrinas (Foster et al.).

\section{TRATAMIENTO DE LA EDAI}

El tratamiento de elección para la deshidratación causada por la diarrea es el reemplazo de líquidos y electrolitos perdidos; por la solución de rehidratación oral o hidratación parenteral, lo que dependerá del grado de deshidratación. Como esta terapia de rehidratación no disminuye significativamente la frecuencia ni la duración de la diarrea, es evidente que se requiere de tratamientos complementarios.

Los episodios de diarrea infecciosa aguda siguen siendo una importante carga de enfermedad en todo el mundo, especialmente en los países en desarrollo. Se deben a la infección por muchos organismos diferentes. 
La mayoría de los episodios son autolimitados y, por lo general, no se realizan investigaciones para identificar el agente infeccioso. El principal riesgo para la salud es la deshidratación y el objetivo del manejo es mejorar y mantener el estado de hidratación. Sin embargo, los líquidos de rehidratación no reducen el volumen de las heces ni acortan el episodio de diarrea.

Probióticos. Se podría señalar de forma simplista, que son "bacterias amigables", que ayudan a mejorar la salud, que no son dañinas en sí mismas; y que son beneficiosos en el tratamiento de la diarrea infecciosa aguda.La definición de la OMS hace referencia a microorganismos vivos que cuando son administrados en cantidad adecuada, ejercen un efecto beneficioso sobre la salud del huésped (Organización de las Naciones Unidas para la Agricultura y la Alimentación, 2006). Según la Asociación Científica Internacional para los Probióticos y Prebióticos (ACIPP), el espectro de los productos y preparaciones que pueden considerarse como probióticos es muy amplio; abarcando desde fármacos probióticos, alimentos de usos médicos especiales con probióticos, como nutrición enteral con probióticos); alimentos probióticos, como leches fermentadas; fórmulas infantiles, como algunas leches en polvo; hasta probióticos de administración no oral, como algunos de administración vaginal). Para ser considerado probiótico es necesario que se hayan realizado estudios en humanos que efectivamente demuestren los beneficios concretos sobre la salud de las cepas concretas (Organización de las Naciones Unidas para la Agricultura y la Alimentación).

Existe evidencia que apoya el beneficio de los probióticos en el tratamiento y prevención de enfermedades en los niños, como en el tratamiento de alergias, cólicos infantiles, enfermedad diarreica aguda, disfunciones gastrointestinales, colon irritable, obesidad, sepsis neotal; y en la prevención de la enterocolitis necrotizante neonatal (Játiva et al., 2016).

Los probióticos incluyen bacterias y levaduras. La mayoría de los probióticos incluyen especies de Lactobacillus, Bifidobacterium, Enterococcus, E. coli, Streptococcus y Lactococcus lactis. La levadura más utilizada es Saccharomyces boulardii. Los probióticos deben sobrevivir al ácido gástrico y la bilis, para que puedan ejercer su efecto en el intestino delgado y grueso. Los probióticos colonizan el intestino temporalmente y actúan modificando el ambiente del colon de acuerdo con la persistencia fecal de las cepas ingeridas. Diferentes mecanismos están involucrados en el papel protector de los probióticos y están representados tanto por la interacción directa con el huésped como por la modulación indirecta de la microbiota intestinal (Gallo et al., 2016).
Evidencias del uso de probióticos en EDAI: En una RS compuesta por 5 ensayos clínicos controlados, cuya población fue de 619 pacientes portadores de EDAI, y un metaanálisis utilizando modelo de efectos aleatorios, se concluyó que la utilización de SB redujo significativamente el tiempo de duración de la diarrea respecto del uso de placebo ($1,1$ días; IC $95 \%:-1,3 ;-0,8)$. Además, se verificó que el uso de SB redujo significativamente el riesgo de diarrea en los días 3,6 y 7. Por otra parte, también se constató que hubo una reducción del riesgo de diarrea de más de 7 días de duración; con un RR de 0,25 (IC 95\%:0,08; 0,83) y un NNT de 5 (IC $95 \%: 3 ; 20$ ). Nivel de evidencia 2a para estudios de tratamiento, grado de recomendación B (Szajewska et al., 2007; Manterola et al., 2014).

En otra RS compuesta por ensayos clínicos aleatorios y cuasialeatorios, que compararon los resultados de la administración de un probiótico vs. un placebo o ningún probiótico; compuesta por 63 estudios, cuya población fue de 8014 lactantes y niños con EDAI; se pudo constatar que los probióticos redujeron la duración de la diarrea, aunque el tamaño del efecto varió considerablemente entre los estudios; observándose además, que el promedio del efecto fue significativo para la duración media de la diarrea (diferencia media 24 horas; IC $95 \%$ : 15,9; 33,6 horas); la diarrea que dura 4 o más días (RR: 0,41; IC $95 \%: 0,32 ; 0.53)$; y la frecuencia de deposiciones al segundo día (diferencia de medias 0,80 ; IC $95 \%$ : 0,45; 1,14 ). Tampoco se atribuyeron eventos adversos a la intervención probiótica. Nivel de evidencia 3 a para estudios de tratamiento, grado de recomendación B (Allen et al.; Manterola et al.).

En otra RS conmeta-análisis compuesta por 19 ensayos clínicos aleatorios, cuya población fue de 1306 pacientes; se verificó que SB redujo la duración de la diarrea, con una diferencia de medias de -0.99 días (IC $95 \%$ : -1,40; $0,58)$; y que SB redujo el riesgo de diarrea durante el tratamiento RR: 0,52 (IC $95 \%$ : 0,42; 0,65). Nivel de evidencia 2a para estudios de tratamiento, grado de recomendación B (Dinleyici et al., 2012; Manterola et al.).

En otra RS, compuesta por 22 estudios, que incluyen 2440 pacientes; el meta-análisis permitió verificar que SB acortó el tiempo de evolución de la diarrea, con una diferencia de medias de 219,7 horas (IC $95 \%$ : 226,1; 213,3); que SB disminuyó la frecuencia de deposiciones al $2^{\circ}$ día, con una diferencia de medias de 20,7 horas (IC $95 \%$ : 21,4; 20,1); y redujo el riesgo de diarrea al $3^{\circ}$ día, con un RR de 0,41 (IC $95 \%: 0,27 ; 0,60$ ) y al $4^{\circ}$ día, con un RR de 0,38 (IC $95 \%$ : $0,24 ; 0,59)$. Nivel de evidencia 2 a para estudios de tratamiento, grado de recomendación B (Feizizadeh et al., 2014; Manterola et al.). 
Otra RS realizada con 24 EC aleatorios o cuasialeatorios, que incluyeron a recién nacidos prematuros $<37$ semanas de edad gestacional o $<2500 \mathrm{~g}$ de peso al nacer, o ambos; permitió constatar en su meta-análisis; que la suplementación con probióticos enterales redujo significativamente la incidencia de enterocolitis necrotizante grave, con un RR de 0,43 (IC $95 \%$ : 0,33; 0,56); y la mortalidad, con un RR de 0,65 (IC $95 \%$ : 0,52; 0,81 ). Nivel de evidencia 2 a para estudios de tratamiento, grado de recomendación B (Alfaleh \& Anabrees, 2014; Manterola et al.).

Por otra parte, en otra RS compuesta por 25 EC, que representan una población de 5868 lactantes prematuros de muy bajo peso al nacer, comparando el uso de probióticos vs. placebo. El meta-análisis realizado utilizando un modelo de efectos fijos, permitió demostrar que el uso de probióticos previene la sepsis de inicio tardío RR de 0,79 (IC $95 \%$ : 0,71; 0,88; NNT de 28). Nivel de evidencia 2 a para estudios de tratamiento, grado de recomendación B (Manterola et al.; Aceti et al., 2017).

En un estudio observacional de la Red Neonatal Alemana,que incluyó 7516 lactantes prematuros menores de 29 semanas de gestación, estratificados según tipo de alimentación (lactancia materna exclusiva, lactancia materna y fórmulas; y sólo fórmulas); se verificó que la exposición del subgrupo de lactancia materna exclusiva a probióticos Lactobacillus acidophilus/ Bifido bacterium infantis, permitió una mejora de las medidas de crecimiento. Nivel de evidencia $3 \mathrm{~b}$ para estudios de tratamiento, grado de recomendación B (Manterola et al.; Fortmann et al. 2020). Hallazgos similares a los obtenidos en un estudio observacional multicéntrico anterior, en el se que estudió el efecto de Lactobacillus acidophilus/Bifido bacterium infantis en 5351 infantes; que permitió verificar una reducción del riesgo de morbilidad gastrointestinal en recién nacidos de muy bajo peso al nacer. Nivel de evidencia $3 \mathrm{~b}$ para estudios de tratamiento, grado de recomendación B (Härtel et al., 2014; Manterola et al.).

Prebióticos. Han sido definidos como ingredientes alimentarios no digeribles que provocan cambios específicos en la composición o actividad del microbiota intestinal confiriendo bienestar y salud al huésped. El Grupo Experto en Prebióticos del International Life Sciences Institute (ILSI), definió la acción prebiótica como la actividad selectiva del crecimiento o actividad de un número limitado de especies microbianas en la microbiota intestinal, que confiere bienestar y salud al huésped (Latulippe et al., 2013). Por su parte, la Word Gastroenterology Organization (WGO), señaló que los prebióticos son sustancias de las dietas especialmente polisacáridos no amiláceos y oligosacáridos no digeribles por enzimas humanas que nutren a grupos seleccionados de microorganismos que habitan en el intestino, favoreciendo el crecimiento de bacterias beneficiosas sobre las nocivas (Guarner et al., 2012).

La microbiota gastrointestinal difiere entre lactantes alimentados con leche materna y fórmula. La leche materna es rica en oligosacáridos prebióticos y también puede contener algunos probióticos, muchas compañías de fórmula infantil a menudo agregan una u otra o ambas. Utilizan en fórmulas infantiles, galacto-oligosacáridos, fructooligosacáridos, polidextrosa y mezclas de estos, pero ninguno agrega oligosacáridos de leche humana. Existe evidencia de que la adición de prebióticos a la fórmula infantil aproxima el microbiota gastrointestinal de los lactantes alimentados con fórmula a la de los lactantes alimentados con leche materna. Los prebióticos cambian la actividad metabólica intestinal, tienen un efecto bifidogénico y acercan la consistencia de las heces y la frecuencia de defecación a las de los lactantes. Se ha determinado efectos beneficiosos y que estos ingredientes son muy seguros acercándose más al estándar dorado de la leche materna (Vandenplas et al., 2015).

Por lo tanto, se han realizado varios estudios con diferentes tipos de fórmulas infantiles enriquecidas con oligosacáridos no digeribles, que se conocen como prebióticos. Los prebióticos típicamente alcanzan el colon intacto y estimulan selectivamente el crecimiento y la actividad de miembros beneficiosos específicos de la microbiota (Wopereis et al.).

Oligosacáridos. Son compuestos polímeros que están formados por 3 y 10 monosacáridos, los cuales se encuentran unidos por enlaces glucosídicos, se reconoce los maltoligosácaridos y los oligosacáridos no digeribles (Cabezas-Zabala et al., 2016).

Por una parte, está la inulina, que está formada por oligosacáridos y polisacáridos. por otra parte, oligosacáridos que se obtienen por hidrólisis de la inulina presente en productos vegetales, o mediante trans-fructosilación enzimática, a partir de sacarosa, utilizando fructosiltransferasas (van de Wiele et al., 2007). Actualmente está aceptado que la inulina y los FOS no se degradan ni se absorben en el tracto gastrointestinal superior, de tal forma que llegan intactos al colon donde son metabolizados por el microbiota intestinal. Diferentes ensayos de fermentación in vitro utilizando cultivos puros de microorganismos o cultivos de heces humanas; así como ensayos en humanos, han puesto de manifiesto que la inulina y los FOS favorecen el crecimiento de bifidobacterias y lactobacilos, disminuyendo el de bacteroides y clostridios (Macfarlane et al.). 
Tanto el Generally Recognized as Safe (GRAS) en EE. UU., como el Foods of Specified Health Use (FOSHU) en Japón; han permitido que inulina y oligofructosa sean utilizados sin restricciones en un gran número de alimentos como yogurts, bebidas, barritas de cereales, galletas, cereales y productos de bollería; o formando parte de alimentos simbióticos. Sus fuentes naturales son: achicoria, cebolla, ajo, alcachofa, yacón, puerro, y espárragos (Hernández Hernández et al.).

Simbióticos. Son preparaciones farmacéuticas o alimentarias que contienen una o más especies de probióticos e ingredientes de prebióticos, correlacionándose entre la actividad de los microorganismos y la metabolización de los prebióticos. Favorecen el desarrollo y actividad de los probióticos, potencian sus propiedades saludables y tendrían un efecto sinérgico. Se espera que esta mejora se acompañe de un aumento cuantitativo de los componentes probióticos (Varela Moreiras et al., 2013).

La disbiosis del ecosistema intestinal contribuye al desarrollo de ciertas enfermedades que pueden ser revertidas por alteraciones favorables de los probióticos. Las alteraciones varían desde diarrea infecciosa y la diarrea asociada a antibióticos, hasta obesidad y trastornos neurológicos. Varios ensayos clínicos controlados se han desarrollado para mostrar efectos biológicos importantes en varias de estas afecciones mediante la administración de prebióticos, probióticos y simbióticos. Los estudios han demostrado que los probióticos alteran la flora intestinal y conducen a la elaboración de metabolitos de la flora que influyen en la salud a través de efectos antimicrobianos directos, mejora de la integridad de la barrera mucosa y modulación inmune.

La administración de probióticos restaura el equilibrio de la flora intestinal para le prevención y tratamiento de varias enfermedades señaladas en acápites anteriores, está claro también que existen diferencias importantes entre las diferentes probióticos. La metagenómica y la meta transcriptomía junto con la bioinformática nos han permitido tener mayor información sobre la acción cruzada entre la microbiota y el huésped, lo que nos permitirá conocer de mejor y profundamente sobre las nuevas generaciones de estos productos biológicos (Patel \& DuPont, 2015).

\section{ESCALA BITSS}

En 2009, se desarrolló y validó la escala Ámsterdam InfantStoolScale (AISS); instrumento de evaluación para consistencia, volumen y color de las heces de niños menores de 1 año (Bekkali et al., 2009) y aunque existe eviden- cia que AISS es más adecuada para lactantes que la escala Bristol Stool Scale (BSFS) (Martinez \& de Azevedo, 2012), en la práctica clínica o la investigación pediátrica; ésta no se utiliza de forma masiva.

Dando respuesta a lo anterior, es que en 2017, se desarrolló en Bruselas, la escala BITSS (Brussels Infant and Toddler Stool Scale) (Vandenplas et al., 2017; Huysentruyt et al., 2019). Esta herramienta de valoración visual de heces adaptada para bebés y niños pequeños que usan pañales, que consta de 7 fotografías en color de pañales que contienen heces de bebés y niños pequeños no entrenados para ir al baño, las que se seleccionaron a través de múltiples sesiones de votación en función de su parecido con el BSFS original según un grupo de enfermeras y médicos. Se determinó validez comparándola con la escala BSFS, mediante 2462 observadores (657 médicos, 624 enfermeras y 1181 padres); obteniéndose un buen nivel de acuerdo general 0,72 (IC $95 \%$ : 0,59; 0,85), con buenos valores $\mathrm{k}$ tanto para médicos, enfermeras y padres; así como para los diferentes centros participantes. Las fotografías BITSS con mejor desempeño correspondieron con BSFS tipo $7(87,5 \%)$ y tipo $4(87,6 \%)$, seguidas de las fotografías de BITSS que representan BSFS tipo $6(75,0$ $\%)$; BSFS tipo 5 (68,0 \%); BSFS tipo 1 (64,8 \%); y BSFS tipo $3(64,6 \%)$. Sobre la base de estos resultados, las fotografías se clasificaron por grupo de heces como heces duras (BSFS tipo 1-3), formadas (BSFS tipo 4), sueltas (BSFS tipos 5 y 6) y acuosas (BSFS tipo 7). De acuerdo con este nuevo sistema de categorización, la asignación correcta para cada fotografía osciló entre $83,0 \%$ y $96,0 \%$. Por lo que se concluyó que la proporción general de asignaciones correctas fue de 72,8 \%; mostrando BITSS un buen nivel de acuerdo con BSFS, por lo que se puede decir que BITSS es una escala confiable entre observadores para la evaluación de las deposiciones de niños sin entrenamiento para ir al baño, para ser aplicada en la práctica clínica e investigación (Huysentruyt et al.).

JÁTIVA-MARIÑO, E.; MANTEROLA, C.; MACIAS, R. \& NARVÁEZ, D. Probiotics and prebiotics. Its role in childhood acute diarrheal disease therapy. Int. J. Morphol., 39(1):294-301, 2021.

SUMMARY: In Ecuador childhood acute diarrheal disease (CADD) constitutes a serious public health problem, representing the 2 nd cause of infant morbidity and mortality in children under 5 years of age. Oral and parenteral hydration in hospitalized children, with standard treatments according to their degree of dehydration and weight loss, as well as preventive measures such as mandatory vaccination against rotavirus, have contributed to a decrease. Nevertheless, this childhood disease has still not been resolved. There are multiple contributing 
factors involved that prevent complete eradication of the disease Among these are socio-economic problems, education, early weaning and poor feeding practices, all of which continue to affect infants. Recent studies have proposed the use of probiotics that help reduce the problem and it has been suggested that Saccharomyces boulardii (SB), associated with a prebiotic, would reduce the treatment time of an CADD. Therefore, the symbiosis between the SB probiotic and a prebiotic called fructooligosaccharide (FOS) could be an alternative to reduce complications and reduce costs. An alternative to measure the clinical course of an CADD in infants is the BITTS scale, which was recently created and can easily be applied by clinicians. The aim of this manuscript was to summarize the existing evidence regarding the role of PROBIOTICS and prebiotics in the treatment of CADD.

KEY WORDS: Sacaccharomyces bourdalii; Prebiotic; Probiotic; Acute diarrheal disease; BITSS scale.

\section{REFERENCIAS BIBLIOGRÁFICAS}

Aceti, A.; Maggio, L.; Beghetti, I.; Gori, D.; Barone, G.; Callegari, M. L.; Fantini, M. P.; Indrio, F.; Meneghin, F.; Morelli, L.; et al. Probiotics prevent late-onset sepsis in human milk-fed, very low birth weight preterm infants: systematic review and meta-analysis. Nutrients, 9(8):904, 2017.

Alfaleh, K. \& Anabrees, J. Probiotics for prevention of necrotizing enterocolitis in preterm infants. Cochrane Database Syst. Rev., (4):CD005496, 2014.

Allen, S. J.; Martinez, E. G.; Gregorio, G. V. \& Dans, L. F. Probiotics for treating acute infectious diarrhoea. Cochrane Database Syst. Rev., 2010(11):CD003048, 2010.

Bekkali, N.; Hamers, S. L.; Reitsma, J. B.; Van Toledo, L. \& Benninga, M. A. Infant stool form scale: development and results. J. Pediatr, 154(4):521-526.e1, 2009.

Bernardo, D. Human intestinal dendritic cells as controllers of mucosal immunity. Rev. Esp. Enferm. Dig., 105(5):279-90, 2013.

Bertelsen, R. J.; Jensen, E. T. \& Ringel-Kulka, T. Use of probiotics and prebiotics in infant feeding. Best Pract. Res. Clin. Gastroenterol., 30(1):39-48, 2016.

Cabezas-Zabala, C. C.; Hernández-Torres, B. C. \& Vargas-Zárate, M. Azúcares adicionados a los alimentos: efectos en la salud y regulación mundial. Revisión de la literatura. Rev. Fac. Med., 64(2):31929, 2016.

Collado, M. C.; Cernada, M.; Neu, J.; Pérez-Martínez, G.; Gormaz, M. $\&$ Vento, M. Factors influencing gastrointestinal tract and microbiota immune interaction in preterm infants. Pediatr. Res., 77(6):726-31, 2015.

Dinleyici, E. C.; Eren, M.; Ozen, M.; Yargic, Z. A. \& Vandenplas, Y. Effectiveness and safety of Saccharomyces boulardii for acute infectious diarrhea. Expert Opin. Biol. Ther, 12(4):395-410, 2012.

Feizizadeh, S.; Salehi-Abargouei, A. \& Akbari, V. Efficacy and safety of Saccharomyces boulardii for acute diarrhea. Pediatrics, 134(1):e176-91, 2014.

Fortmann, I.; Marißen, J.; Siller, B.; Spiegler, J.; Humberg, A.; Hanke, K.; Faust, K.; Pagel, J.; Eyvazzadeh, L.; Brenner, K.; et al. Lactobacillus acidophilus/bifidobacterium infantis probiotics are beneficial to extremely low gestational age infants fed human milk. Nutrients, 12(3):850, 2020.
Foster, J. A. \& McVey Neufeld, K. A. Gut-brain axis: how the microbiome influences anxiety and depression. Trends Neurosci., 36(5):305-12, 2013.

Foster, J. A. Gut microbiome and behavior: focus on neuroimmune interactions. Int. Rev. Neurobiol., 131:49-65, 2016.

Foster, J. A.; Rinaman, L. \& Cryan, J. F. Stress \& the gut-brain axis: Regulation by the microbiome. Neurobiol. Stress, 7:124-36, 2017.

Gaci, N.; Borrel, G.; Tottey, W.; O'Toole, P. W. \& Brugère, J. F. Archaea and the human gut: new beginning of an old story. World $J$. Gastroenterol., 20(43):16062-78, 2014.

Gallo, A.; Passaro, G.; Gasbarrini, A.; Landolfi, R. \& Montalto, M. Modulation of microbiota as treatment for intestinal inflammatory disorders: An uptodate. World J. Gastroenterol., 22(32):7186-202, 2016.

Grönlund, M. M.; Grzes`kowiak, ?.; Isolauri, E. \& Salminen, S. Influence of mother's intestinal microbiota on gut colonization in the infant. Gut Microbes, 2(4):227-33, 2011.

Guarner, F.; Khan, A. G.; Garisch, J.; Eliakim, R.; Gangl, A.; Thomson, A.; Krabshuis, J.; Lemair, T.; Kaufmann, P.; de Paula, J. A.; et al. World Gastroenterology Organisation Global Guidelines: probiotics and prebiotics October 2011. J. Clin. Gastroenterol., 46(6):468-81, 2012.

Härtel, C.; Pagel, J.; Rupp, J.; Bendiks, M.; Guthmann, F.; RiegerFackeldey, E.; Heckmann, M.; Franz, A.; Schiffmann, J. H.; Zimmermann, B.; et al. Prophylactic use of Lactobacillus acidophilus/ Bifidobacterium infantis probiotics and outcome in very low birth weight infants. J. Pediatr., 165(2):285-289.e1, 2014.

Hernández Hernández, A.; Coronel Rodríguez, C.; Monge Zamorano, M. \& Quintana Herrera, C. Microbiota, probióticos, prebióticos y simbióticos. Pediatr. Integr., 19(5):337-54, 2015.

Huysentruyt, K.; Koppen, I.; Benninga, M.; Cattaert, T.; Cheng, J.; De Geyter, C.; Faure, C.; Gottrand, F.; Hegar, B.; Hojsak, I.; et al. The Brussels Infant and Toddler Stool Scale: a study on interobserver reliability. J. Pediatr. Gastroenterol. Nutr., 68(2):207-13, 2019.

Instituto Nacional de Estadística y Censos (INEC). Informe de Rendición de Cuentas. Período: enero - diciembre 2017. Quito, Instituto Nacional de Estadística y Censos, 2017. Disponible en: https:// www.ecuadorencifras.gob.ec/documentos/web-inec/ Rendicion_de_cuentas_2017/Fase_3/Informe \%20tecnico \%20Planta \%20Central \%20RC \%202017(final)10FEB2018.pdf

Játiva, E.; Velasco-Benítez, C. A.; Koppen, I. J. N.; Játiva-Cabezas, Z. \& Saps, M. Prevalence of functional gastrointestinal disorders in schoolchildren in Ecuador. J. Pediatr. Gastroenterol. Nutr., 63(1):258, 2016.

Khanna, S. \& Tosh, P. K. A clinician's primer on the role of the microbiome in human health and disease. Mayo Clin. Proc., 89(1):107-14, 2014

Latulippe, M. E.; Meheust, A.; Augustin, L.; Benton, D.; Berc`^̂́k, P.; Birkett, A.; Eldridge, A. L.; Faintuch, J.; Hoffmann, C.; Miller Jones, J.; et al. ILSI Brazil International Workshop on Functional Foods: a narrative review of the scientific evidence in the area of carbohydrates, microbiome, and health. Food Nutr. Res., 57, 2013.

Macfarlane, G. T.; Steed, H. \& Macfarlane, S. Bacterial metabolism and health-related effects of galacto-oligosaccharides and other prebiotics. J. Appl. Microbiol., 104(2):305-44, 2008.

Manterola, C.; Asenjo-Lobos, C. \& Otzen, T. Hierarchy of evidence: levels of evidence and grades of recommendation from current use. Rev. Chilena Infectol., 31(6):705-18, 2014.

Martinez, A. P. \& de Azevedo, G. R. Traducción, adaptación cultural y validación de la "Bristol Stool Form Scale". Rev. Latinoam. Enferm., 20(3):583-9, 2012.

Montrose, D. C. \& Floch, M. H. Probiotics used in human studies. $J$. Clin. Gastroenterol., 39(6):469-84, 2005. 
Organización de las Naciones Unidas para la Agricultura y la Alimentación (FAO/OMS). Probióticos en los Alimentos. Propiedades Saludables y Nutricionales y Directrices para la Evaluación. Estudio FAO Alimentación y Nutrición 85. Roma, Organización de las Naciones Unidas para la Agricultura y la Alimentación, 2006. Disponible en: http://www.fao.org/3/a-a0512s.pdf

Patel, R. \& DuPont, H. L. New approaches for bacteriotherapy: prebiotics, new-generation probiotics, and synbiotics. Clin. Infect. Dis., $60 \mathrm{Suppl}$. 2(Suppl. 2):S108-21, 2015.

Sanz, Y.; Collado, M. C. \& Dalmau, J. Contribución de la microbiota intestinal y del género "Bifidobacterium" a los mecanismos de defensa del huésped frente a patógenos gastrointestinale. Acta Pediatr. Esp., 64(2):74-8, 2006.

Szajewska, H.; Skórka, A. \& Dylag, M. Meta-analysis: Saccharomyces boulardii for treating acute diarrhoea in children. Aliment. Pharmacol. Ther., 25(3):257-64, 2007.

van de Wiele, T.; Boon, N.; Possemiers, S.; Jacobs, H. \& Verstraete, W. Inulin-type fructans of longer degree of polymerization exert more pronounced in vitro prebiotic effects. J. Appl. Microbiol., 102(2):45260, 2007.

Vandenplas, Y.; Szajewska, H.; Benninga, M.; Di Lorenzo, C.; Dupont, C.; Faure, C.; Miqdadi, M.; Osatakul, S.; Ribes-Konickx, C.; Saps, M.; et al. Development of the Brussels Infant and Toddler Stool Scale ('BITSS'): protocol of the study. BMJ Open, 7(3):e014620, 2017.

Vandenplas, Y.; Zakharova, I. \& Dmitrieva, Y. Oligosaccharides in infant formula: more evidence to validate the role of prebiotics. Br. J. Nutr., 113(9):1339-44, 2015.

Varela Moreiras, G. (Ed.). Libro Blanco de la Nutrición en España. $3^{\mathrm{a}}$ ed. Madrid, Fundación Española de la Nutrición (FEN), 2013. Disponible en: https://www.seedo.es/images/site/ documentacionConsenso/Libro_Blanco_Nutricion_Esp-2013.pdf

Vitetta, L.; Vitetta, G. \& Hall, S. Immunological tolerance and function: associations between intestinal bacteria, probiotics, prebiotics, and phages. Front. Immunol., 9:2240, 2018.

Wopereis, H.; Sim, K.; Shaw, A.; Warner, J. O.; Knol, J. \& Kroll, J. S. Intestinal microbiota in infants at high risk for allergy: Effects of prebiotics and role in eczema development. J. Allergy Clin. Immunol., 141(4):1334-1342.e5, 2018.

Zamudio-Vázquez, V. P.; Ramírez-Mayans, J. A.; Toro-Monjaraz, E. M.; Cervantes-Bustamante, R.; Zárate-Mondragón, F.; Montijo-Barrios, E.; Cadena-León, J. F. \& Cázares-Méndez, J. M. Importance of gastrointestinal microbiota in children. Acta Pediatr. Mex., 38(1):4962, 2017.

\author{
Dirección para correspondencia : \\ Dr. Carlos Manterola Delgado \\ Departamento de Cirugía y CEMyQ \\ Universidad de La Frontera \\ Manuel Montt 112 Oficina 408 \\ Temuco-CHILE
}

Email: carlos.manterola@ufrontera.cl

Recibido : 19-08-2020

Aceptado: 11-09-2020 